\title{
La Influencia del WhatsApp en la Educación Superior de la UNACAR
}

\section{The Influence of WhatsApp in Higher Education of the UNACAR}

\author{
Dámaris Pérez-Cruz', Fabian Sánchez-López², José Felipe Cocón-Juárez y y Patricia Zavaleta-Carrillo
}

\section{() \\ EDICIÓN: @e-CIVTAC}

Recibido: 6/julio/2020

Aceptado: 5/agosto/2020

Publicado: 25/septiembre/2020

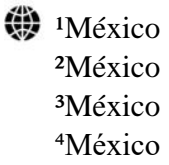

\section{IIIInstitución}

${ }^{1}$ Universidad Autónoma del Carmen ${ }^{2}$ Universidad Autónoma del Carmen ${ }^{3}$ Universidad Autónoma del Carmen ${ }^{4}$ Universidad Autónoma del Carmen

\section{Correo Eletrónico}

1dperez@pampano.unacar.mx 21410918@mail.unacar.mx 3jfcocon@hotmail.com

[4pzavaleta@pampano.unacar.mx

\section{(DORCID}

1https://orcid.org/0000-0002-6226-9561 ${ }^{2}$ https://orcid.org/0000-0001-9340-5709 ${ }^{3} \mathrm{https} / / /$ orcid.org/0000-0002-6932-683X ${ }^{4} \mathrm{https}: / /$ orcid.org/0000-0003-2001-6950

\section{Citar así: CGAPA / IEEE}

Pérez-Cruz, D., Sánchez-López, F., CocónJuárez, J. \& Zavaleta-Carrillo, P. (2020). La Influencia del WhatsApp en la Educación Superior de la UNACAR. Revista Tecnológica-Educativa Docentes 2.0, 9(2), 39-48. https://doi.org/10.37843/rted.v9i2.143

D. Pérez-Cruz, F. Sánchez-López, J. Cocón-Juárez y P. Zavaleta-Carrillo, "La Influencia del WhatsApp en la Educación Superior de la UNACAR", RTED, vol. 9, n. ${ }^{\circ} 2$, pp. 39-48, sep. 2020.

\section{Resumen}

Actualmente, la masificación de los teléfonos celulares, así como de las aplicaciones para ellos, se han hecho presentes en diferentes sectores de la sociedad. Entre las aplicaciones de mensajería instantánea ha destacado el WhatsApp que se ha hecho parte de la vida diaria de la mayoría de las personas que cuentan con un celular. La investigación se centró en el uso del WhatsApp, para conocer la influencia que tiene entre los estudiantes de la Universidad Autónoma del Carmen (UNACAR). El objetivo de esta investigación fue saber, primero que tanto utilizan el WhatsApp, y segundo que tanto es útil para sus actividades académicas. Se realizó una investigación cuantitativa, sobre un tema innovador y que está presente en la vida cotidiana, el WhatsApp y su uso entre estudiantes de educación superior. Para el estudio se usó una encuesta que se aplicó a 100 estudiantes. Los resultados obtenidos demostraron el uso intensivo de dicha aplicación. El WhatsApp puede llegar a ser una herramienta de comunicación efectiva entre estudiantes y profesores, debido al alcance que tiene el uso de la herramienta entre las personas, la mayoría cuenta con un teléfono inteligente, el cual seguramente tiene la aplicación de WhatsApp instalada, aunque habrá que considerar que existen otras aplicaciones que posiblemente tengan mejores funcionalidades que el WhatsApp. Por los hallazgos encontrados en la investigación realizada se concluye que el WhatsApp es una herramienta ampliamente usada entre estudiantes de nivel superior.

Palabras clave: Educación superior, internet, aplicaciones, WhatsApp, mensajería instantánea.

\begin{abstract}
Currently, the spread of cell phones, as well as applications for them, have been present in different sectors of society. Among the instant messaging applications, WhatsApp has stood out, which has become part of the daily life of most people who have a cell phone. The research focused on the use of WhatsApp, to know the influence it has among the students at the Autonomous University of Carmen (UNACAR). The objective of this research was to know, first how much they use WhatsApp, and second how much it is useful for their academic activities. A quantitative research was carried out, on an innovative topic that is present in everyday life, the WhatsApp, and its use among higher education students. For the study, a survey was applied to 100 students. The results obtained demonstrated the intensive use of said application. WhatsApp can become an effective communication tool between students and teachers, due to the scope of the use of the tool among people, most have a smartphone, which surely has the WhatsApp application installed, although there will be What to consider that there are other applications that possibly have better functionalities than WhatsApp. Based on the findings of the research carried out, it is concluded that WhatsApp is a widely used tool among higher level students.
\end{abstract}

Keywords: Higher Education, internet, applications, WhatsApp, instant messaging. 
La comunicación, según la RAE (2014), acción y efecto de comunicar o comunicarse; trato, correspondencia entre dos o más personas; es más que la acción que por su significado y la intención de quien comunica se convierte en un mensaje (LópezJiménez, 2007). En este sentido, la mensajería instantánea es un servicio de comunicación síncrona, es decir, comunicación en tiempo real entre dispositivos, ya sean computadoras de escritorio o portátiles, tabletas, celulares, entre otros. La mensajería instantánea ha evolucionado desde los 90's, hasta nuestros días, se ha sofisticado y adoptado como parte del uso cotidiano (Castro, 2019).

Por consiguiente, la aplicación de mensajería instantánea es sin duda alguna uno de los grandes desarrollos del siglo XXI. Aunque, la mensajería instantánea ya existía desde hace varios años en el entorno informático, pero no fue sino con la masificación de los teléfonos inteligentes o smartphones que se logra concebir llevar de esa manera la comunicación en el bolsillo (Cahun, 2018). Recientemente, Amaia (Arribas-Urrutia, 2019) publica que existe una penetración de Internet del 66.6\% para América Latina y el Caribe para una población de $658^{\prime} 345,820$. Por ende, más del $50 \%$ cuenta con acceso a Internet, siendo también usuarios de Facebook y de aplicaciones de mensajería instantánea (Arribas-Urritia, Islas-Carmona, \& Gutiérrez-Cortés, 2019).

Recientes publicaciones de (Jiménez-Pitre et al., 2019, p. 179) indican que las redes sociales más populares entre los niños y jóvenes en orden decreciente son: WhatsApp, Instagram, Telegram, Snapchat, Facebook y un muy pequeño número utiliza Imo, por lo que la red más utilizada es WhatsApp. El servicio de WhatsApp es una plataforma de mensajería síncrona más usada en todo el mundo por más de mil millones de personas al día, en varios países se posiciona como la aplicación móvil más utilizada, ya que en su gran mayoría se cuenta con un dispositivo móvil, con la aplicación de mensajería instantánea.

En este mismo sentido, WhatsApp utiliza una conexión a Internet bien sea a través de datos o red Wifi institucional, gubernamental o doméstica (Portilla, 2020). Ahora, centrándose un poco en el pasado, verán como surgirán algunas dudas acerca de la comunicación en el pasado, verán como surgirán algunas dudas acerca de la comunicación en el pasado, hay que preguntarse ¿Cómo se comunicaban?, si analizamos esto con precisión pueden darse cuenta de que se ha avanzado mucho tecnológicamente hablando como sociedad el día de hoy, además, de que se sigue innovando y creando cosas totalmente nuevas.

El presente artículo busca analizar cómo influye la aplicación de mensajería instantánea WhatsApp en los estudiantes del nivel superior. Se espera que este trabajo sea de gran aporte a la comunidad universitaria, para que facilite la llegada de avisos, notificaciones personales, actividades o tareas de escolares, considerando las decisiones del alumno a través de la aplicación de mensajería instantánea ya mencionada. Esta aplicación ha tomado gran relevancia en la universidad hoy en día por pandemia, por tanto, surgieron ciertas curiosidades sobre el aprovechamiento de WhatsApp en los jóvenes universitarios y en la nueva forma de comunicación de enseñanza-aprendizaje que se vive en la Universidad Autónoma del Carmen (UNACAR).

\section{Breve Historia de la Comunicación y de WhatsApp}

La comunicación humana según (MáximaUriarte, 2020) "es una categoría compleja y amplia, en la que caben numerosas formas de intercambio de información y numerosos lenguajes posibles, verbales o no, y a través de canales diferentes" (p.1). Asimismo, el autor interpreta que "la comunicación nació con el ser humano, mismo ya que es una de sus capacidades naturales" (p.2).

En este mismo orden de ideas, Mendoza (2013) describe que las primeras muestras de comunicación fueron primordialmente los gestos y sonidos culturales, y posteriormente, se fueron desarrollando algunas habilidades, como la comunicación que se comenzó a transmitir a través de imágenes, hasta la comunicación con el apoyo tecnológico. Logrando potenciar los medios de comunicación, incrementando la difusión cultural y el uso masivo de los medios digitales hoy en día.

El autor Romero (2020) sustenta que WhatsApp fue fundada por Acton \& Koum en 2009, después de dejar su trabajo en Yahoo!. Todo inició cuando dejaron su trabajo para viajar alrededor del mundo. Pero pronto sus ahorros comenzaron a hundirse, tuvieron que solicitar un trabajo en Facebook, que tampoco funcionó según lo planeado. 
Estaban bastante decepcionados, pero este fracaso los llevó a comenzar un nuevo viaje de su vida: WhatsApp. En un mundo donde la demanda y popularidad de una aplicación nunca son estables, Acton junto con Koum (Pahwa, 2020, p.1) lograron mantener su oferta en la cima a lo largo de la historia de WhatsApp; gracias a su estrategia de no anuncios. En lugar de perseguir el dinero interrumpiendo continuamente los chats con anuncios, dieron más prioridad al interés del usuario, que es lo que más cuenta hoy en día.

Más de 1.500 millones de personas en 180 países usan WhatsApp hoy. Esta plataforma ya se hizo cargo de SMS, MMS, además de otras características obsoletas que su mercado objetivo utilizó anteriormente para comunicarse entre sí. Ahora, con la visión de Facebook de incluso incorporar empresas, estas funciones de los operadores móviles podrían terminar siendo completamente inútiles.

Zuckerberg no deja piedra sin mover para llevar a WhatsApp a un nuevo nivel en el mercado empresarial. Se trata de una nueva versión de escritorio de la aplicación (WhatsApp Web) para facilitar su uso en una PC o Mac (Pahwa, 2020, p.2). No obstante, Jan Koum había lanzado WhatsApp con un propósito ser una mensajería de poco peso, a bajo coste, al menos en principio comentado por (Romero, 2020, p.1).

Por otra parte, Internet como las aplicaciones de los dispositivos móviles y de la propia red se irán innovando con el paso del tiempo, es algo inevitable, cada día se van creando nuevas infraestructuras tecnológicas, tanto de software, como de hardware. Tal como como sucedió con la aplicación para móviles WhatsApp, misma que en 2009, según Adriana (Salinas, 2017) ya estaba operando en los teléfonos móviles.

Finalmente, el logotipo de WhatsApp consta de una nube de conversación con un teléfono levantado en el centro, el verde significa que la línea de comunicación está constantemente abierta, se puede llamar a cualquier lugar, como lo menciona (TICbeat, 206, p.1). Cabe resaltar, el color verde del ícono el cual es utilizado como una bandera para transmitir positivismo, además de transmitir mayor confianza entre los consumidores de este tipo de aplicaciones (1000marcas.net, 2020).

\section{Aparición de WhatsApp Web}

WhatsApp Web se lanzó en 2015 la cual permite utilizar WhatsApp en una computadora de escritorio o portátil, sincronizando el teléfono con la computadora mediante un código QR (CascónBaños, 2016), (Collado, 2020). En otras palabras, WhatsApp Web puede ejecutarse en cualquier computadora a través de un navegador (browser), tal como, Opera, Firefox, Chrome o Safari) a través de cualquier computadora, así se menciona en Michán (2015) y Bravo (2020).

Una vez que se entra al WhatsApp Web, a simple vista se verá que la pantalla se divide en dos zonas principales. En el lado izquierdo encontramos el listado de todos los contactos, mientras que en el lado derecho aparecerán las conversaciones de manera individual del contacto seleccionado. Además, de enviar y recibir mensajes de la misma manera que lo hacemos a través de la aplicación de WhatsApp en el teléfono móvil, se puede enviar distintos tipos de archivos como fotos, vídeos y documentos alojados en nuestra computadora, y también modificar el estado, perfil y demás. Es posible descargar en la computadora cualquier tipo de archivo como fotos, vídeos o documentos asociados a WhatsApp del teléfono móvil con solo seleccionar el archivo y ordenar la descarga, todo de manera muy sencilla.

También se pueden compartir enlaces de sitios web seleccionados anteriormente en el navegador con los contactos. Se debe recordar que para hacer uso de esta herramienta web se utiliza el ratón o el techado de la computadora, ya sea de escritorio (desktop) o computadora portátil (laptop), para muchas personas esto es una comodidad. Esta herramienta es una aplicación segura desarrollada por los mismos creadores de WhatsApp. Siempre se debe tener presente, que para que WhatsApp Web funcione correctamente el teléfono móvil deberá estar encendido, conectado a Internet y cercano a la computadora o portátil (ACDesDigital, 2019).

\section{Metodología}

Esta investigación fue de corte cuantitativa que de acuerdo con los autores (Hernández-Sampieri et al., 2014) y por las características de esta metodología, nos permite planteamientos acotados, mide fenómenos, utiliza estadística, entre otras, además, de que entre sus bondades nos permite la generación de resultados, control sobre fenómenos, precisión y predicción, requeridos para llevar a cabo 
el desarrollo de la investigación.

En este mismo sentido, ante el uso de WhatsApp en la universidad, se plantea, lo siguiente, se puede evaluar la influencia del WhatsApp en los estudiantes de la toda la universidad, abarcando una muestra de la población estudiantil de nivel superior. Considerando, los planteamientos anteriores, se formula el siguiente objetivo principal de esta investigación: conocer sobre la influencia del WhatsApp en el ámbito académico de los estudiantes del nivel superior en la Universidad Autónoma del Carmen (UNACAR). Evaluar frecuencia de uso y el tiempo dedicado a la aplicación Whats App solamente en los estudiantes del nivel superior de la UNACAR. Por otra parte, mediante investigación documental bibliográfica, se encontraron las ventajas $\mathrm{y}$ desventajas del uso de la aplicación WhatsApp.

Para medir estos fenómenos en este estudio, se sustenta con la realización de encuestas que permiten recopilar los datos, así obtener resultados, realizar un análisis estadístico mediante herramientas computacionales para dar una respuesta con más precisión, tratando de cumplir con los objetivos planteados. La obtención de los datos se recopiló de una población de estudiantes del nivel superior al azar. Siendo que los estudiantes del nivel superior en general suelen usar WhatsApp con mayor frecuencia, para poder comunicarse con sus profesores, compañeros, ya sea para tarea o avisos importantes.

Para la población encuestada, se contó con participación de estudiantes de los diferentes semestres, de los diferentes programas educativos con los que cuenta la Universidad Autónoma del Carmen (UNACAR) del campus principal de Ciudad del Carmen, ya que la UNACAR tiene varios campus en distintos lugares. En este mismo orden de idea, las encuestas se aplicarán a 100 estudiantes.

Un paso de gran relevancia en este trabajo fue diseñar el instrumento de encuesta a utilizar, para después crearla a través de una plataforma para diseñar formularios en línea denominado Google Forms, dónde a través de un enlace o enlace que proporciona se puede acceder al formulario de encuesta, en la figura 1 se puede observar el enlace mencionado. La encuesta está formada por diez preguntas de opción múltiple dirigidas a los estudiantes del nivel superior revisada de acuerdo con los objetivos de la investigación.

\section{Figura 1}

Enlace para acceder a la encuesta de Google Forms.

\section{Enviar formulario}

Recopilar direcciones de correo electrónico

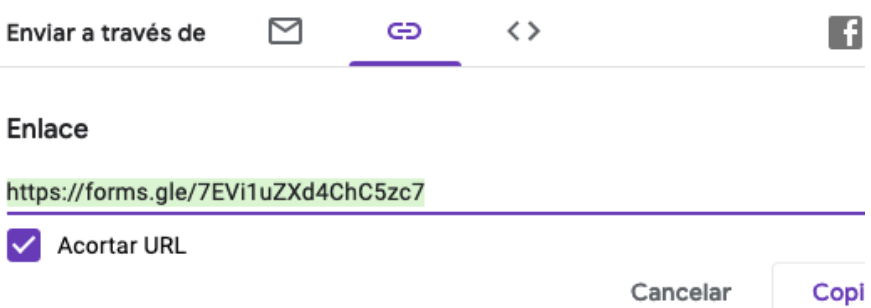

Nota. Enlace final hacia el formulario elaborado y publicado en la herramienta de Google Forms. Fuente: Herramienta de Google Forms.

\section{Instrumento}

El instrumento denominado Encuesta uso de WhatsApp, muestra el título "Influencia del WhatsApp en la educación superior", a continuación, se da una breve explicación de lo que trata el instrumento, posteriormente se solicitan los datos del estudiante encuestado, solo se solicitan dos datos al estudiante: sexo y semestre en el que se encuentra, aparte de ello, se encuentra la lista de preguntas relacionadas con el uso del WhatsApp (ver figura 2).

Cómo se observa en la figura 2, la encuesta está diseñada con escala de tres puntos o tres niveles, con la intención de medir de manera más subjetiva cada pregunta, aunque la escala de cinco puntos o cinco niveles (Matas, 2018) propone medir la neutralidad según la escala de Likert (Shum-Xie, 2020), (Bedoya-Laguna, 2017), en este caso particular, al definir elementos precisos y cerrados, permite evaluar de forma más clara y directa cada uno de los elementos.

Con escala de tres puntos, dado el instrumento, en primera, se obtiene de respuesta: Si, No, Tal vez o Algunas veces; en segundo, se obtiene una respuesta: Bastante, Poco o Regular; finalmente en alguna pregunta se obtiene de respuesta: Siempre, Algunas veces o nunca, siendo la primera respuesta con mayor valor en el instrumento, y así, respectivamente. En algunos casos, las respuestas cerradas limitan las respuestas de los encuestados con el fin de obtener una respuesta apegada a la realidad de los jóvenes universitarios, tal es el caso, como lo que se obtiene de respuesta a: 1-2 horas, 
3-4 horas o No lo utilizo, ya que son las frecuencias de uso en tiempo utilizado por los estudiantes, considerado en una reunión con el experto.

\section{Figura 2}

\section{Cuestionario de la encuesta}

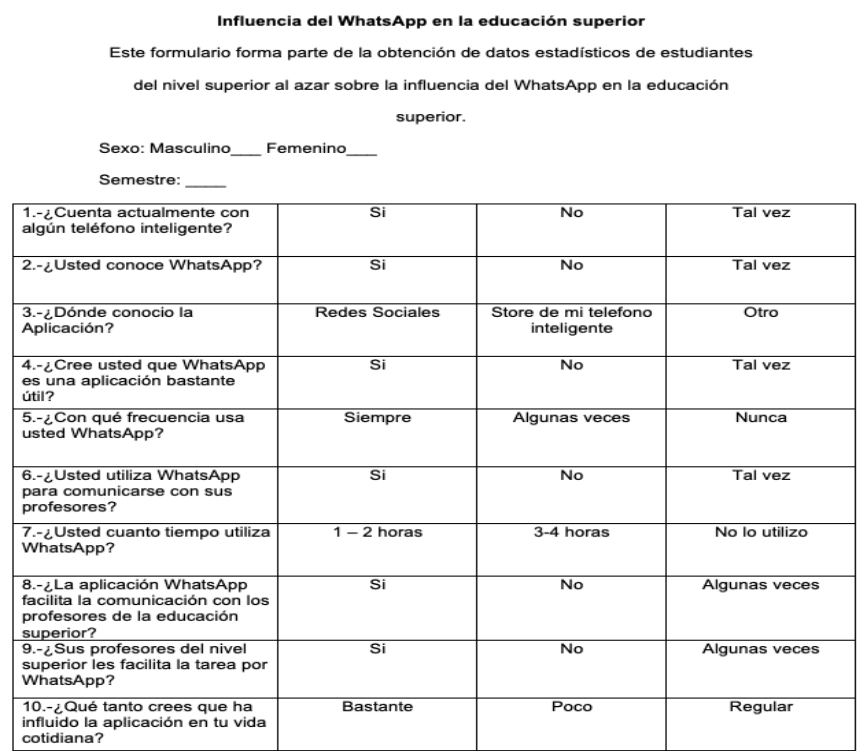

Nota. Muestra las diez preguntas realizadas a los estudiantes de nivel superior, elaboración propia de los autores.

\section{Resultados}

Durante un periodo de tres meses dentro del Campus principal de la UNACAR se entrevistó de manera aleatoria a 100 estudiantes a los que se les solicito que ingresarán a la página indicada por la liga o enlace de la encuesta y la contestarán. Posteriormente, se obtuvo del Google Forms, el archivo con las respuestas recabadas de las encuestas se procedió a analizar la información, generando un reporte con gráficas y estadísticas de las respuestas de todas las preguntas, por un lado, en formato general incluyendo a todas las preguntas, y por el otro, en formato individual para cada una de las preguntas, finalmente, dar difusión a los resultados obtenido.

En la tabla 1 se puede observar la frecuencia de respuestas dadas para cada una de las opciones asociadas con las preguntas de las encuestas, expresando en porcentaje dicha frecuencia, en general en cada una de las preguntas el mayor porcentaje se refleja en la primera opción de la pregunta y esta opción corresponde, para la mayoría de las preguntas a la parte afirmativa del uso de la aplicación. Para facilitar la visualización de las respuestas en las opciones de cada una de las preguntas en la figura 3 se presentan solo los porcentajes de las preguntas con la gráfica correspondiente a estos porcentajes, dentro de la cual se puede observar que la opción uno es la de mayor frecuencia en las respuestas obtenidas.

\section{Tabla I}

Resultados en porcentaje de la encuesta aplicada a los alumnos del nivel superior.

\begin{tabular}{|c|c|c|c|}
\hline Opción & Opción 1 & Opción 2 & $\begin{array}{c}\text { Opción } \\
3\end{array}$ \\
\hline $\begin{array}{l}\text { 1. ¿Cuenta } \\
\text { actualmente } \\
\text { con algún } \\
\text { teléfono } \\
\text { inteligente? }\end{array}$ & $\begin{array}{l}\mathrm{Si} \\
96 \%\end{array}$ & $\begin{array}{l}\text { No } \\
0 \%\end{array}$ & $\begin{array}{c}\text { Tal vez } \\
4 \%\end{array}$ \\
\hline $\begin{array}{ll}\text { 2. ¿Usted conoce } \\
\text { WhatsApp? }\end{array}$ & $\begin{array}{l}\mathrm{Si} \\
96 \%\end{array}$ & $\begin{array}{l}\text { No } \\
1 \%\end{array}$ & $\begin{array}{c}\text { Tal vez } \\
3 \%\end{array}$ \\
\hline $\begin{array}{l}\text { 3. ¿Dónde conoció } \\
\text { la aplicación? }\end{array}$ & $\begin{array}{l}\text { Redes } \\
\text { sociales } \\
29 \%\end{array}$ & $\begin{array}{l}\text { Store de mi } \\
\text { teléfono } \\
\text { inteligente } \\
16 \% \\
\end{array}$ & $\begin{array}{l}\text { Otro } \\
55 \%\end{array}$ \\
\hline $\begin{array}{l}\text { 4. ¿Cree usted que } \\
\text { WhatsApp es } \\
\text { una aplicación } \\
\text { bastante útil? }\end{array}$ & $\begin{array}{l}\mathrm{Si} \\
72 \%\end{array}$ & $\begin{array}{l}\text { No } \\
2 \%\end{array}$ & $\begin{array}{c}\text { Tal vez } \\
26 \%\end{array}$ \\
\hline $\begin{array}{ll}\text { 5. ¿Con que } \\
\text { frecuencia usa } \\
\text { usted } \\
\text { WhatsApp? }\end{array}$ & $\begin{array}{l}\text { Siempre } \\
38 \%\end{array}$ & $\begin{array}{l}\text { Algunas } \\
\text { veces } \\
61 \%\end{array}$ & $\begin{array}{c}\text { Nunca } \\
1 \%\end{array}$ \\
\hline $\begin{array}{l}\text { 6. ¿Usted utiliza } \\
\text { WhatsApp para } \\
\text { comunicarse } \\
\text { con sus } \\
\text { profesores? }\end{array}$ & $\begin{array}{l}\mathrm{Si} \\
75 \%\end{array}$ & $\begin{array}{l}\text { No } \\
9 \%\end{array}$ & $\begin{array}{c}\text { Tal vez } \\
16 \%\end{array}$ \\
\hline $\begin{array}{l}\text { 7. ¿Usted cuánto } \\
\text { tiempo utiliza } \\
\text { WhatsApp? }\end{array}$ & $\begin{array}{l}1-2 \text { horas } \\
57 \%\end{array}$ & $\begin{array}{l}\text { 3-4 horas } \\
41 \%\end{array}$ & $\begin{array}{l}\text { No lo } \\
\text { utilizo } \\
2 \%\end{array}$ \\
\hline $\begin{array}{l}\text { 8. ¿La aplicación } \\
\text { WhatsApp } \\
\text { facilita la } \\
\text { comunicación } \\
\text { con los } \\
\text { profesores de la } \\
\text { educación } \\
\text { superior? }\end{array}$ & $\begin{array}{l}\mathrm{Si} \\
53 \%\end{array}$ & $\begin{array}{l}\text { No } \\
14 \%\end{array}$ & $\begin{array}{c}\text { Algunas } \\
\text { veces } \\
33 \%\end{array}$ \\
\hline $\begin{array}{ll}\text { 9. ¿Sus profesores } \\
\text { de nivel } \\
\text { superior les } \\
\text { facilita la tarea } \\
\text { por WhatsApp? }\end{array}$ & $\begin{array}{c}\mathrm{Si} \\
31 \%\end{array}$ & $\begin{array}{l}\text { No } \\
13 \%\end{array}$ & $\begin{array}{c}\text { Algunas } \\
\text { veces } \\
56 \%\end{array}$ \\
\hline $\begin{array}{l}\text { 10. ¿Qué tanto } \\
\text { crees que ha } \\
\text { influido la } \\
\text { aplicación en tu } \\
\text { vida cotidiana? }\end{array}$ & $\begin{array}{l}\text { Bastante } \\
37 \%\end{array}$ & $\begin{array}{l}\text { Poco } \\
27 \%\end{array}$ & $\begin{array}{c}\text { Regular } \\
36 \%\end{array}$ \\
\hline
\end{tabular}

Nota. Respuestas en porcentaje de manera general de las preguntas aplicadas por los estudiantes. Fuente: Elaboración propia de los autores. 


\section{Figura 3}

Resultados de la encuesta aplicada a los alumnos del nivel superior.

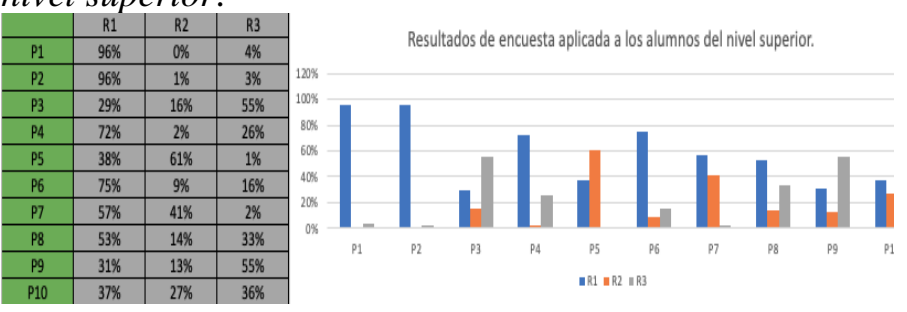

Nota. Gráfico y resultados de cada una de las respuestas empleadas para cada pregunta, producto del cuestionario o encuesta aplicada. Fuente: Elaboración propia de los autores.

\section{Tabla 2}

Resultados por género de los alumnos del nivel superior.

\begin{tabular}{|l|c|}
\hline Género & Encuestados \\
\hline Hombres & 53 \\
\hline Mujeres & 47 \\
\hline
\end{tabular}

Nota. Se muestra el total de 100 estudiantes encuestados.

\section{Tabla 3}

Resultado del semestre que cursan los alumnos del nivel superior.

\begin{tabular}{|c|c|}
\hline Semestre & Estudiantes \\
\hline 2 & 24 \\
\hline 4 & 26 \\
\hline 6 & 25 \\
\hline 8 & 25 \\
\hline
\end{tabular}

Nota. Se nuestra la cantidad de estudiantes de los diferentes semestres en la que actualmente están cursando los estudiantes.

En este sentido, se muestran los resultados de las tablas 2 y 3 , ya que son los primeros registros de la encuesta, en dónde se puede comentar que se demuestra una equidad de género en los encuestados (Tabla 2). En este mismo sentido, en la tabla 3, el rango de encuestados es muy similar, indicando que se tiene un rango de muestra con respecto al semestre cursado, es decir, se muestra los diferentes semestres que existen en el momento de la aplicación del formulario de encuesta. Al analizar cada uno de los resultados a cada pregunta, los alumnos fueron muy claro con sus respuestas, así como los comentarios de acuerdo con que tanto influye o es importante el uso de WhatsApp en nivel de educación superior.
La primera pregunta: ¿Cuenta actualmente con algún teléfono? De los cien estudiantes encuestados, el $96 \%$ de los alumnos del nivel superior cuentan con un teléfono inteligente para su uso cotidiano, el $4 \%$ contesto que tal vez tenían un teléfono, demostrando que toda la comunidad estudiantil encuestada de nivel superior actualmente cuente con un teléfono inteligente, sin considerar la gama del teléfono, como se puede observar en el gráfico (Figura 4).

\section{Figura 4}

Gráfico de estudiantes que cuentan con teléfono inteligente.

$$
100 \text { respuestas }
$$

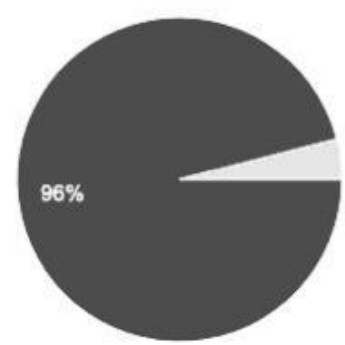

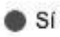

No Tal vez
Nota. En el gráfico describe el $96 \%$ con respuesta afirmativa, $0 \%$ con respuesta negativa, el $4 \%$ con respuesta Tal vez tienen un teléfono inteligente.

En la siguiente pregunta: ¿Usted conoce WhatsApp? Cómo se puede observar en gráfico (Figura 5), el 96\% de los alumnos tienen conocimiento de WhatsApp para móviles, 3 contestaron que tal vez conozcan, mientras que 1 alumno desconoce totalmente del WhatsApp. Se puede confirmar el hecho de que mayormente los estudiantes que tienen un teléfono inteligente también tienen instalada una aplicación de mensajería instantánea, tal como el WhatsApp.

\section{Figura 5}

Gráfico de alumnos del nivel superior que conocen WhatsApp

100 respuestas
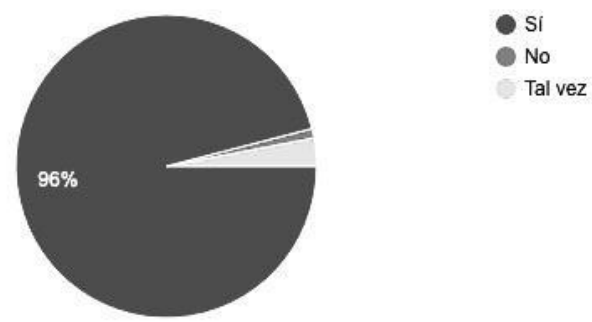

Nota. En el gráfico describe el $96 \%$ con respuesta afirmativa, $1 \%$ con respuesta negativa, el 3\% con respuesta Tal vez, que los estudiantes conocen el WhatsApp. 
La pregunta: ¿Cree usted que WhatsApp es una aplicación útil? El $72 \%$ de los alumnos del nivel superior cree que WhatsApp es una aplicación bastante útil, tanto a nivel académico como en la vida cotidiana, el $26 \%$ de los alumnos dijo que tal vez podría ser muy útil pero que algo no los convence, esperemos que se terminen enamorando de WhatsApp, mientras que el otro $2 \%$ dice que no les es útil y les parece una aplicación muy ventajosa, como se puede observar en la gráfica que se presenta (Figura 6).

\section{Figura 6}

Gráfico de alumnos del nivel superior sobre la utilidad de WhatsApp.

100 respuestas
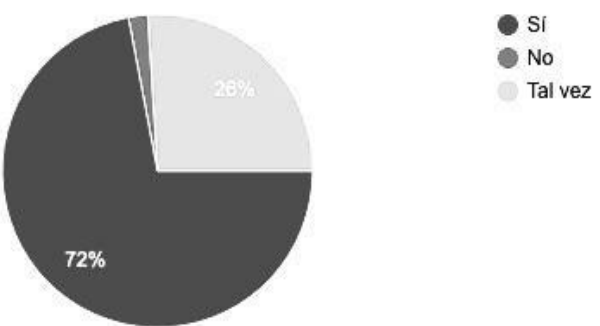

Nota. En el gráfico se describe el $72 \%$ con respuesta afirmativa, $26 \%$ con respuesta negativa, el $2 \%$ con respuesta Tal vez, sobre la utilidad de WhatsApp.

En la pregunta: ¿Usted utiliza WhatsApp para comunicarse con sus profesores? Como se observa en el gráfico de la figura 7 , el $75 \%$ de los alumnos mantienen contacto o se comunican con su profesor por medio de WhatsApp, $16 \%$ de los alumnos tal vez se comunican con sus profesores por medio de WhatsApp, y el 9\% restante dice que no mantienen contacto con sus profesores por WhatsApp.

\section{Figura 7}

Gráfico de alumnos que se comunican con sus profesores por medio de WhatsApp.

100 respuestas
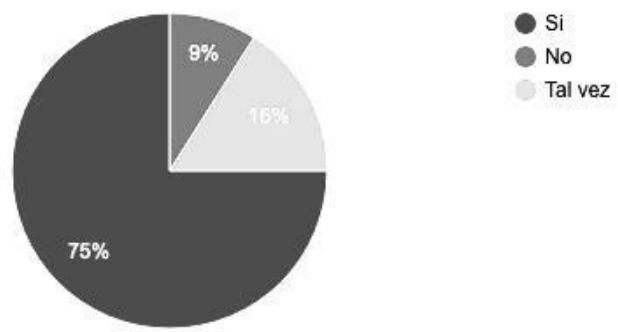

Nota. En el gráfico se describe el $75 \%$ con respuesta afirmativa, $16 \%$ con respuesta negativa, el $9 \%$ con respuesta Tal vez, sobre la comunicación con los profesores a través de WhatsApp.
En el siguiente cuestionamiento: ¿La aplicación WhatsApp facilita la comunicación con los profesores de la educación superior? Como se ve puede observar en el gráfico (Figura 8), un poco más de la mitad de los alumnos dice que WhatsApp les facilita la comunicación con su profesor, mientras que $33 \%$ de los alumnos de educación superior indica que, si se les ha facilitado la comunicación algunas veces, más no todo el tiempo, y el $14 \%$ restante dice que no les facilita para nada la aplicación.

\section{Figura 8}

Gráfico de alumnos que se les facilita la comunicación con sus profesores.

100 respuestas

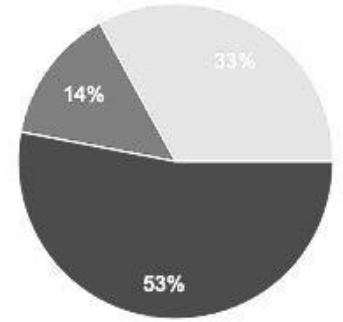

$\mathrm{Si}$

No

Algunas veces

Nota. En el gráfico se describe el $53 \%$ con respuesta afirmativa, $14 \%$ con respuesta negativa, el 33\% con respuesta Algunas veces, que se les facilita la comunicación con sus profesores.

Finalmente, el penúltimo cuestionamiento de la encuesta: ¿Sus profesores del nivel superior les facilita la tarea por WhatsApp? En el gráfico (Figura 9), se muestra que al $56 \%$ de los alumnos del nivel superior algunas veces su profesor les manda tarea por medio del WhatsApp, 31\% dijo que sus profesores si les facilita tarea por medio del WhatsApp, y el otro $13 \%$ dice que sus profesores no les mandan tarea.

\section{Figura 9}

Gráfico de alumnos a los que sus profesores les facilita la tarea por WhatsApp.

100 respuestas
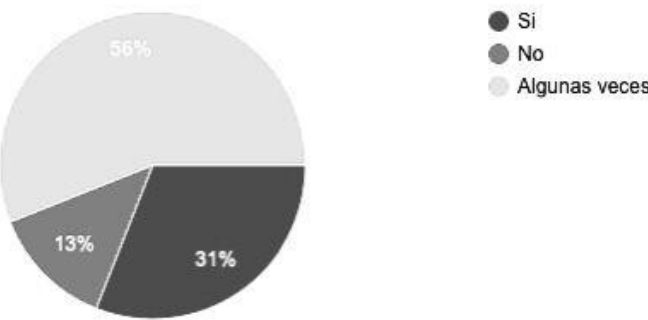

Nota. En el gráfico se describe el $31 \%$ con respuesta afirmativa, $13 \%$ con respuesta negativa, el 55\% con respuesta Algunas veces, que el profesor les facilita la tarea por WhatsApp. 
Ventajas y Desventajas del WhatsApp en la Educación Superior

Como todo existen ventajas, desventajas del uso de WhatsApp, las escuelas necesitan una aplicación móvil para comunicarse, así como mantenerse en contacto con los estudiantes. Tener una forma estructurada de comunicación. Sin embargo, los centros escolares no pueden desarrollar una aplicación móvil escolar u obtener un software de gestión escolar debido al factor costo, licenciamiento, tiempo, entre otras. Aunque se enfrentarían al mantenimiento de dicha aplicación, las actualizaciones periódicas, corrección de errores, control de versiones, entre otras. En su lugar, eligen plataformas de chat para mantenerse en contacto (ONNE CREW, 2019, p.1).

Hoy, WhatsApp es utilizado activamente por todo tipo de personas por múltiples razones. Entre ellos, están los adolescentes, que gobiernan la base activa de usuarios. WhatsApp es su lugar de referencia, desde la planificación hasta el intercambio de información y notas de clase. Sin embargo, WhatsApp también tiene sus contras. Cómo lo comenta (Choundhury, 2019, p.1) en su artículo.

Sin embargo, en este estudio se listan las ventajas, desventajas de la aplicación WhatsApp en la educación superior, son las siguientes:

\section{Ventajas:}

1. Aplicación fácil de usar para enviar fotos, videos, documentos al instante, entre otras.

2. Estupenda para el uso personal.

3. Puedes recuperar mensajes perdidos.

4. Obtener respuesta instantánea o en tiempo real.

5. Es gratuito.

\section{Desventajas:}

1. No es muy ordenada (Fotos dentro de los chats, chats, videos dentro de los chats, entre otros).

2. Por ser gratuito tiene limitaciones.

3. Múltiples grupos creados pueden crear mucha confusión.

4. Es difícil obtener información exacta, por ejemplo, cantidad de alumnos que respondieron $\mathrm{Si}$ o No u otra respuesta, para dar seguimiento a una información solicitada.

\section{Discusiones}

El análisis anteriormente mostrado se llevó a cabo en los alrededores de la Universidad Autónoma del Carmen, es decir, dentro del campus principal; se ofrecieron encuestas a los alumnos del nivel superior, ciertos alumnos creen que WhatsApp es una aplicación que ha cobrado gran relevancia en el nivel educativo, ya que gracias a ella se les facilitan ciertas acciones. Comentaban que les es fácil que un profesor le haga llegar algún aviso sobre una clase o actividad dentro del curso, además de que algunas veces les comparte información de la clase, ya sea libros para estudiar y/o tareas. A continuación, se resume en tres categorías los resultados de los estados de esta investigación:

1. Alcance. Se evidencia que la influencia del WhatsApp en el nivel superior ha sido grande, ya que brinda facilidades para el envío y recepción de avisos, dudas, envío de libros, imágenes, pequeños vídeos, explicaciones en audio, enlaces a sitios web, creación de grupos de trabajo, grupos de difusión, llamadas, videollamadas. Además, de contar con las versiones de escritorio y de navegador web que ofrece mayor utilidad al WhatsApp por su diversificación de opciones.

2. Tiempo. Con respecto al WhatsApp, se tiene un tiempo de respuesta casi instantáneo al momento de enviar un mensaje, con la facilidad de conocer si el mensaje le ha sido entregado, de igual modo si el mensaje ha sido leído. Con respecto a la investigación, se requirió mucha dedicación tanto para la investigación de campo, como para la aplicación de encuestas, asimismo, con el diseño y elaboración de este.

3. Costos. En cuanto al WhatsApp, no requiere ningún pago, para la versión estándar, por lo que tanto profesores como alumnos pueden descargar y acceder de manera gratuita la aplicación, sin embargo, sí se requiere contar con datos para utilizarla. En la investigación, monetariamente hablando, no se requirió inversión, dado que las herramientas utilizadas son gratuitas, tan sólo mucha dedicación para alcanzar los objetivos y paciencia para la recopilación y procesamiento de las encuestas.

\section{Conclusión}

Siempre es bueno aprender acerca de las cosas como lo fue en esta ocasión el software de aplicación 
WhatsApp, el cual tuvo un gran revuelo a lo largo de los años; así como en la vida cotidiana de las personas, que incluso se empezó a utilizar en la educación superior, para facilitar exactamente la comunicación con las personas de alrededor, sea cual sea el momento o la circunstancia, el software de aplicación WhatsApp tiene sus lados positivos, como la de facilitar los materiales didácticos, avisos de los profesores a los alumnos o viceversa, entre otras.

Al igual que cualquier aplicación tiene sus desventajas, pero lo más notable es no poder comunicarse si no se tienen datos o wifi, pero al final de cuentas no deja de ser útil en las escuela o en la vida cotidiana, pues hoy en día ¿Quién no tiene un teléfono? o ¿Quién no tiene acceso a Internet?, hoy en día fácilmente se tiene acceso a muchas cosas en la red, en el cual se le puede sacar mucho provecho para nuestras actividades cotidianas.

Se concluye que esta investigación demuestra la clara influencia del software de aplicación WhatsApp en la comunidad estudiantil de la UNACAR, quedando evidenciado en la última pregunta del cuestionario: ¿Qué tanto crees que ha influido la aplicación en tu vida cotidiana? Con un $73 \%$ con regular o bastante y un $27 \%$ poco, es decir, claramente demostrado que el uso del software de aplicación WhatsApp es parte fundamental en los jóvenes universitarios.

Por otro lado, la contingencia actual causada por el virus SARS-CoV-2 ha requerido el uso intensivo de las comunicaciones síncronasasíncronas, es decir, se ha recurrido al uso de las aplicaciones de software que ofrecen las nuevas tecnologías de los dispositivos actuales, como son el WhatsApp y WhatsApp Web. Permitiendo comunicación constante con los estudiantes, a bajo costo y sin requerir grandes recursos de hardware, sin embargo, aún existe una brecha de aprendizaje en la comunidad docente para la explotación de esta tecnología.

\section{Agradecimientos}

Expresamos nuestro agradecimiento a los estudiantes de nivel superior, que participaron en la encuesta aplicada vía on-line (en línea).

\section{Referencias}

ACDesDigital. (2019). ACDesDigital. Recuperado de: https://acdesdigital.org/whatsapp-web-caracteristicasutilidades/

Arribas-Urritia, A., Islas-Carmona, O., \& Gutiérrez-Cortés, F. (2019). De prosumidores a observadores: una tendencia emergente en Internet y en los jóvenes ecuatorianos. Resultados del estudio del World Internet Project, Ecuador. Revista Latina de Comunicación Social (72), 969-996. doi:10.4185/RLCS-20191367

Arribas-Urrutia, A. (2019). La brecha digital. Recuperado de: http://dspace.uhemisferios.edu.ec:8080/xmlui/handle/12345678 9/1069

Bedoya-Laguna, C. A. (2017). Diseño de un instrumento tipo escala Likert para la descripción de las actitudes hacia la tecnología por parte de los profesores de un colegio público de Bogotá. Bogotá, Colombia: Universidad Distrital Francisco José de Caldas. Recuperado de: http://repository.udistrital.edu.co/bitstream/11349/6881/1/Bedo yaLagunaCrihstianAlberto2017.pdf

Bravo, M. (2020). Fayer Wayer. Recuperado de: https://www.fayerwayer.com/2020/03/whatsapp-web-trucosinternet/

Cahun, A. (2018). XATAKA Recuperado de: https://www.xataka.com.mx/aplicaciones/asi-nacio-whatsapp

Cascón-Baños, J. (2016). Frikipandi. Recuperado de https://www.frikipandi.com/moviles/20160629/historiawhatsapp/

Castro, L. (2019). About español. Recuperado de: https://www.aboutespanol.com/que-es-im-o-mensajeriainstantanea-y-como-funciona-157567

Choundhury, S. (2019). Grabon. Recuperado de: https://blog.grabon.in/advantage-disadvantage-whatsapp/

Collado, C. (2020). Andro4all. Recuperado de: https://andro4all.com/guias/whatsapp/whatsapp-web-pc-tabletmovil

Real Academia Española (2014). Comunicación. La 23. a edición. Recuperado de: https://dle.rae.es/comunicaci\%C3\%B3n

Hernández-Sampieri, R., Fernández-Collado, C., \& Baptista-Lucio, M. (2104). Metodología de la investigación. McGraw-hill

Jiménez-Pitre, I. A., Jiménez-Pitre, N. L., \& Molina-Bolivar, G. (2019). Proceso de aprendizaje en niños y adolescentes potencializado a través de las redes sociales. Revista Tecnológica-Educativa Docente 2.0, 7(2), 168-181. Recuperado de https://ojs.docentes20.com/index.php/revistadocentes20/article/view/78

López-Jiménez, D. F. (2007). La naturaleza de las tecnologías de información y comunicación: las TIC como determinantes de las organizaciones y de la sociedad de la información. Palabra Clave, 10(1), 72-93. Recuperado de: http://dspace.uhemisferios.edu.ec:8080/xmlui/handle/12345678 9/453 
Matas, A. (2018). Diseño del formato de escalas tipo Likert: un estado de la cuestión. Revista electrónica de investigación educativa, 20(1), 38-47. doi:10.24320/redie.2018.20.1.1347

Máxima-Uriarte, J. (2020). La historia de la comunicación humana. Recuperado de: https://www.caracteristicas.co/historia-de-lacomunicacion-humana/

Mendoza, I. (2013). Utel blog Universidad. Recuperado de: https://www.utel.edu.mx/blog/10-consejos-para/historia-delos-medios-de-comunicacion/

Michán, M. (2015). APPLESFERA. Recuperado de: https://www.applesfera.com/aplicaciones-ios-1/whatsapp-webque-es-como-funciona-y-como-sacarle-el-mejor-partido

ONNE CREW. (2019). ONNE. Recuperado de: http://blog.onne.world/pros-cons-of-using-whatsapp-for-yourschool/

Pahwa, A. (2020). FEEDOUGH. Recuperado de: https://www.feedough.com/history-of-whatsapp/

Portilla, D. (10 de abril de 2020). Herramientas de una aplicación móvil de mensajería instantánea para el fortalecimiento del proceso enseñanza - aprendizaje del inglés. Revista TecnológicaEducativa Docentes 2.0, 9(1), 116-123. doi: https://doi.org/10.37843/rted.v9i1.109

Romero, J. (2020). Trecebits. Recuperado de: https://www.trecebits.com/2020/02/15/cual-es-el-origen-dewhatsapp-por-que-se-llama-whatsapp/

Salinas, A. (2017). Mott marketing. Recuperado de: https://mott.marketing/informacion-sobre-que-es-quien-creoy-como-funciona-la-aplicacion-whatsapp/

Shum-Xie, Y. (2020). Qué es, cómo se usa, dónde se utiliza. Recuperado de: https://yiminshum.com/escala-likertinvestigacion/

TICbeat. (2016). TICbeat. Recuperado de: https://www.ticbeat.com/cyborgcultura/el-significado-de-loslogotipos-de-whatsapp-twitter-o-airbnb/2/ 